\title{
Association of NT-proBNP and Subsequent Major Adverse Cardiovascular and Cerebral Events in Patients with Successful PCI for ACS and Normal LVEF
}

\section{Jia-Li Wang}

Beijing Friendship Hospital, Capital Medical University

Shu-Mei Zhao

Beijing Friendship Hospital, Capital Medical University

\section{Hui Chen}

Beijing Friendship Hospital, Capital Medical University

\section{Chun-Yan Guo}

Beijing Friendship Hospital, Capital Medical University

\section{Xue-Qiao Zhao}

University of Washington

Hong-Wei Li ( $\square$ lhw19656@sina.com )

Capital Medical University Affiliated Beijing Friendship Hospital https://orcid.org/0000-0001-59007088

\section{Research}

Keywords: N-Terminal pro-brain natriuretic peptide (NT-proBNP), acute coronary syndromes (ACS), left ventricular ejection fraction (LVEF), major adverse cardiovascular and cerebral event (MACCE)

Posted Date: July 2nd, 2020

DOI: https://doi.org/10.21203/rs.3.rs-38337/v1

License: (c) (i) This work is licensed under a Creative Commons Attribution 4.0 International License. Read Full License 


\section{Abstract}

Background: Does N-Terminal pro-brain natriuretic peptide (NT-proBNP) predict subsequent major adverse cardiovascular and cerebral event (MACCE) in patients received successful percutaneous coronary intervention (PCl) for acute coronary syndrome (ACS) and had normal left ventricular ejection fraction (LVEF)?

Methods: 3986 ACS patients were divided into 4 groups based on the quartile (Q) values of peak NTproBNP measured during hospitalization. All patients were followed for MACCE, a composite of all-cause death, non-fatal myocardial infarction (MI) or stroke, and heart failure requiring hospitalization (HFRH), during a median of 35 months. The incidence of MACCE was compared among Q1-Q4. Receiver operation characteristic curves (ROC) were generated to compare the area under the curve (AUC) for MACCE, cardiovascular (CV) death and HFRH by adding NT-proBNP to the TIMI (thrombolysis in myocardial infarction) risk score.

Results: The incidences of MACCE $(5.6 \%, 9.1 \%, 13.0 \%, 20.1 \%, P<0.001)$, all-cause death $(1.0 \%, 2.5 \%$, $4.1 \%, 8.4 \%, P<0.001)$, non-fatal MI $(2.0 \%, 3.4 \%, 4.8 \%, 6.2 \%, P<0.001)$ and HFRH $(1.5 \%, 2.3 \%, 4.1 \%, 5.9 \%, P$ $<0.001)$ were significantly increased from Q1 to Q4, but, not stroke $(1.4 \%, 1.4 \%, 1.3 \%, 2.1 \%, P=0.438)$. Each median level $(337 \mathrm{pg} / \mathrm{ml})$ increase in NT-proBNP was significantly and independently associated with increased risk of MACCE (HR 1.02, 95\% Cl, 1.01-1.03; $P<0.001)$. Compared with TIMI (thrombolysis in myocardial infarction) risk score alone, TIMI+NT-proBNP showed improved AUCs: CV death (0.76 vs. 0.72 , $P=0.0008)$, and HFRH (0.68 vs. $0.66, P=0.0017)$, MACCE ( 0.70 vs. $0.69, P=0.0012)$, respectively.

Conclusion: NT-proBNP was significantly and independently associated with increased risk of subsequent MACCE in 3 years in ACS patients who received successful PCI and had normal LVEF, and improved the prognosis of major adverse events in addition to the TIMI risk score.

\section{Background}

Despite the continued advancements in modern treatment strategies including percutaneous coronary intervention (PCI) and medical therapies for coronary artery disease, acute coronary syndrome (ACS) remains to be the leading cause of death worldwide. Major efforts have been made for risk stratification with attempt to guide the early aggressive therapies in the high-risk patient population ${ }^{1,2}$.In addition, reduced left ventricular ejection fraction (LVEF) by echocardiography (echo) is well established independent predictor associated with poor clinical outcomes post $\mathrm{ACS}^{3-5}$. However, LVEF does not provide information on myocardial remodeling caused by the ischemic condition and reperfusion injury before reaching abnormal systolic function post $\mathrm{ACS}^{6}$.

$\mathrm{N}$-Terminal pro-brain natriuretic peptide (NT-proBNP) has been considered as a useful biomarker for assessing myocardial remodeling condition ${ }^{7,8}$.Elevated NT-proBNP as response to ventricular pressure overload and ventricular wall stretch ${ }^{9}$ is associated with development of heart failure and poor clinical 
outcomes ${ }^{10}$.A number of studies have demonstrated that NT-proBNP value was a predictor of clinical outcomes in ACS patients ${ }^{11,12}$. However, the prognostic value of NT-proBNP in patients with successful PCI for ACS and normal LVEF has not been fully investigated. The present study was designed to: (1) examine whether NT-proBNP is associated with myocardial ischemic injury and inflammation and (2)determine whether NT-proBNP at the time of ACS predicts subsequent major adverse cardiovascular and cerebral events (MACCE) in the patients with successful PCl and normal LVEF, therefore, to evaluate the prognostic value of adding NT-proBNP to the existing TIMI (thrombolysis in myocardial infarction) risk score ${ }^{2}$ for subsequent MACCE in this population.

\section{Methods}

\section{Study population}

A total of 6023 ACS patients, who were admitted to Beijing Friendship Hospital and underwent PCI between January 2013 and June 2018, were enrolled into the retrospective observational cohort study. ACS including ST-segment elevation myocardial infarction (STEMI), non-ST-segment elevation myocardial infarction (NSTEMI) and unstable angina pectoris (UAP) was diagnosed and confirmed based on symptoms, electrocardiogram (ECG) changes, cardiac biomarkers, and results of coronary angiography ${ }^{13}$. The decision of $\mathrm{PCl}$ treatment was made by two interventional cardiologists simultaneously consistent with the international standards and guideline ${ }^{14}$.

Patients were included consecutively if they met the 3 following criteria: (1) ACS patients $\geq 18$ years of age. (2) patients with definite diagnosis of ACS and successful PCI procedure. Successful PCI was defined as the attainment of a residual vessel diameter stenosis $\leq 20 \%$ and normal epicardial coronary flow (TIMI-3 flow), and without overt complication and ischemic symptom at discharge. (3) patients with LVEF $\geq 50 \%$ assessed by echo during hospitalization. As shown in Figure 1, 1336 cases were excluded due to missing data, 510 were removed for $\mathrm{LVEF}<50 \%$, and 191 were excluded for the medical history of prior heart failure (HF).

Finally, 3986 patients were included and classified into 4 groups based on peak NT-proBNP quartile values during hospitalization, as group Q1, Q2, Q3, Q4, in this analysis. The study protocol was approved by Institutional Ethics Committee of Beijing Friendship Hospital, and the study was also in accordance with the 1964 Declaration of Helsinki and its later amendments or comparable ethical standards.

\section{Data collection}

Patient demographic information, medical history, cardiovascular risk factors, laboratory assessments, medical therapy for ACS and PCI procedures were collected and recorded in the Cardiovascular Center Beijing Friendship Hospital Database Bank (CBD Bank).In addition to the routine biochemistry testing, serum NT-proBNP, cardiac troponin I (cTnI), creatine kinase-MB (CK-MB) were measured at the initial 
admission and monitored during hospitalization for ACS. The peak value of each of these measurements was selected and used in the current study.

Echocardiography, both M-mode and two-dimensional echo, was performed (Philips IE33) after admission for routine assessments of heart structure and function post ACS. We included LVEF, left atrium diameter (LAD), and left ventricular end-diastolic diameter (LVEDD) in the current analysis.

Coronary angiography was performed at the time of PCI for ACS for identifying the culprit lesion and assessing results of $\mathrm{PCl}$. It also provided an overall evaluation of coronary stenosis severity in the nonculprit levels. A number of epicardial main coronary arteries with stenosis $\geq 50 \%$ were determined for each patient.

\section{Study endpoints and follow-up}

Composite MACCE including all-cause death, non-fatal MI, stroke, and HF requiring hospitalization (HFRH) was defined as the primary study endpoint. The secondary endpoints involved cardiovascular (CV) death and each component of the MACCE mentioned above. CV death was defined as death from $\mathrm{MI}, \mathrm{HF}$, or documented cardiac sudden death. Stroke was referred to new ischemic stroke, confirmed by symptoms and results of computed tomography (CT) scan. Non-fatal MI and HF were determined by symptom, physical sign, results of ECG and echo, and the value of biomarkers. All adverse cardiac events were reviewed and confirmed by two separate cardiologists. After discharge, regular follow-up was completed by clinic visits or by phone interviews every 3-6 months up to June 2019 with a median followup duration of 35 months (IQR: 23,48$)$.

\section{Statistical analysis}

Continuous variables were expressed as a mean \pm SD (standard deviation) or median with IQR (interquartile range) and were compared with the Mann-Whitney $U$ test. Categorical data were expressed as numbers and percentages and were compared by Chi-square or Fisher's exact statistics. Spearman's correlation coefficients were calculated to assess the relationship between log [NT-proBNP] (logtransformed NT-proBNP) and LVEF, LVEDD, peak cTnl, and hs-CRP. The primary and secondary endpoints were compared among the 4 groups respectively by Chi-square test.

Survival analysis was performed using Kaplan-Meier method with the log-rank test. Univariable and multivariable Cox proportional hazards analysis were used to estimate the hazard ratios (HR) and 95\% confidence intervals $(\mathrm{Cl})$. Multivariable Cox regression models with stepwise forward approach to include confounding variables identified from the univariate analysis were used to identify the independent risk predictors of the primary endpoint.

Receiver operating characteristic (ROC) curves were generated for predictive accuracy of subsequent MACCE post successful PCI for ACS. The area under the curve (AUC) was calculated using TIMI risk score and NT-proBNP added to TIMI risk score. Then, AUCs were compared between TIMI risk score and NTproBNP added to TIMI risk score for predictive accuracy of MACCE. 
A two-sided $P$ value $\mathbb{0} 0.05$ was considered to be statistically significant. All statistical analyses were performed using the SPSS software version 23.0(IBM, Armonk, NY, USA)

\section{Results}

\section{Clinical characteristics}

Of 3986 patients enrolled in this study, the mean age was $64 \pm 11$ years, and $70 \%$ was male. As described in Table 1, 1.5\% $(n=2092)$ of the study patients had UAP, $22 \%(n=863)$ had NSTEMI and $26 \%(n=1031)$ had STEMI. The median level of NT-proBNP was 337 with IQR $(111,1164) \mathrm{pg} / \mathrm{ml}$, and the median values of NT-proBNP in the Q1-Q4 groups were 56, 190, 607, $2706 \mathrm{pg} / \mathrm{ml}$. 
Table 1

Clinical characteristics of the cohort grouped by the quartiles of NT-proBNP

\begin{tabular}{|c|c|c|c|c|c|}
\hline Variable & $\begin{array}{l}\text { Q1 } \\
(n=999)\end{array}$ & $\begin{array}{l}\text { Q2 } \\
(n=996)\end{array}$ & $\begin{array}{l}\text { Q3 } \\
(n=996)\end{array}$ & $\begin{array}{l}\text { Q4 } \\
(n=995)\end{array}$ & $\begin{array}{l}P \\
\text { value }\end{array}$ \\
\hline \multicolumn{6}{|l|}{ Demographic } \\
\hline Age, years & $60.3 \pm 8.5$ & $64.1 \pm 10.4$ & $63.2 \pm 11.4$ & $67.7 \pm 11.6$ & $\varangle 0.001$ \\
\hline Male, $\%$ & $755(75.6)$ & $690(69.3)$ & 724 (72.7) & $619(62.2)$ & $\varangle 0.001$ \\
\hline $\begin{array}{l}\text { Body mass index, } \\
\mathrm{kg} / \mathrm{m}^{2}\end{array}$ & $26.3 \pm 3.2$ & $26.0 \pm 3.3$ & $25.8 \pm 3.5$ & $25.4 \pm 3.6$ & $\nabla 0.001$ \\
\hline \multicolumn{6}{|l|}{ Past medical history } \\
\hline Hypertension, n (\%) & $675(67.6)$ & 715 (71.8) & $651(65.4)$ & $653(65.6)$ & 0.007 \\
\hline $\begin{array}{l}\text { Diabetes mellitus, } \mathrm{n} \\
(\%)\end{array}$ & $362(36.2)$ & $354(35.5)$ & $329(33.0)$ & $341(34.3)$ & 0.450 \\
\hline Dyslipidemia, n (\%) & $507(50.8)$ & $471(47.3)$ & $449(45.1)$ & $449(45.1)$ & 0.036 \\
\hline Prior stroke, n (\%) & $134(13.4)$ & $163(16.4)$ & $162(16.3)$ & $143(14.4)$ & 0.177 \\
\hline Smoking, n (\%) & 418 (41.9) & 381 (38.3) & 455 (45.7) & 391 (39.3) & 0.004 \\
\hline Clinical diagnosis & & & & & $\otimes 0.001$ \\
\hline UAP, n (\%) & $899(90.0)$ & 707 (71) & $365(36.6)$ & $121(12.2)$ & $\otimes 0.001$ \\
\hline NSTEMI, n (\%) & $65(6.5)$ & $181(18.2)$ & $324(32.5)$ & $293(29.4)$ & $\otimes 0.001$ \\
\hline STEMI, n (\%) & $35(3.5)$ & $108(10.8)$ & 307 (30.8) & $581(58.4)$ & \\
\hline Length of stay (days) & $5(4-6)$ & $6(5-8)$ & $7(5-8)$ & $8(7-10)$ & $\nabla 0.001$ \\
\hline \multicolumn{6}{|l|}{ Initial presentation } \\
\hline Heart rate, beats/min & $68(63-77)$ & $68(62-76)$ & $70(62-78)$ & $72(64-82)$ & $\nabla 0.001$ \\
\hline Systolic BP, mmHg & $130 \pm 16$ & $133 \pm 18$ & $132 \pm 22$ & $129 \pm 23$ & $\nabla 0.001$ \\
\hline Diastolic BP, mmHg & $77 \pm 11$ & $76 \pm 11$ & $75 \pm 12$ & $73 \pm 12$ & $\varangle 0.001$ \\
\hline Laboratory finding & & & & & \\
\hline
\end{tabular}

NT-proBNP, N-Terminal pro-brain natriuretic peptide; BP, blood pressure; STEMI, ST-elevation myocardial infarction; NSTEMI, non-ST-segment elevation myocardial infarction; UAP, unstable angina pectoris; LDL-C, low-density lipoprotein cholesterol; cTNI, cardiac troponin I; CK-MB, creatine kinaseMB; hs-CRP, high-sensitivity C-reactive protein; LAD, left atrium diameter; LVEDD, left ventricular enddiastolic diameter; ACEl, angiotensin-converting enzyme inhibitors, LVEF, left ventricular ejection fraction; ARB, angiotensin receptor blockers.

*P2Y12 receptor antagonist within 12 months after $\mathrm{PCI}$ 


\begin{tabular}{|c|c|c|c|c|c|}
\hline Variable & $\begin{array}{l}\text { Q1 } \\
(n=999)\end{array}$ & $\begin{array}{l}\text { Q2 } \\
(n=996)\end{array}$ & $\begin{array}{l}\text { Q3 } \\
(n=996)\end{array}$ & $\begin{array}{l}\text { Q4 } \\
(n=995)\end{array}$ & $\begin{array}{l}P \\
\text { value }\end{array}$ \\
\hline $\begin{array}{l}\text { Peak NT-proBNP, } \\
\text { ng/L }\end{array}$ & $56(35,83.2)$ & $190(146,254)$ & $\begin{array}{l}607 \\
(445,838.8)\end{array}$ & $\begin{array}{l}2706(1715, \\
5065)\end{array}$ & $\varangle 0.001$ \\
\hline $\begin{array}{l}\text { Fasting glucose, } \\
\mathrm{mmol} / \mathrm{L}\end{array}$ & $\begin{array}{l}5.51(4.88- \\
6.63)\end{array}$ & $\begin{array}{l}5.54 \\
(4.85,6.79)\end{array}$ & $\begin{array}{l}5.63 \\
(4.85,7.16)\end{array}$ & $\begin{array}{l}5.90(5.03- \\
7.66)\end{array}$ & $\bigotimes 0.001$ \\
\hline Hemoglobin A1c, \% & $6.1(5.6-7.0)$ & $6.1(5.6,7.0)$ & $6.0(5.6,7.1)$ & $6.0(5.5-7.0)$ & 0.567 \\
\hline $\begin{array}{l}\text { Total cholesterol, } \\
\mathrm{mmol} / \mathrm{L}\end{array}$ & $4.29 \pm 1.08$ & $4.31 \pm 1.07$ & $4.44 \pm 1.09$ & $4.45 \pm 1.06$ & $\nabla 0.001$ \\
\hline LDL-C, mmol/L & $2.40 \pm 0.73$ & $2.44 \pm 0.76$ & $2.55 \pm 0.80$ & $2.58 \pm 0.78$ & $\nabla 0.001$ \\
\hline Triglyceride, $\mathrm{mmol} / \mathrm{L}$ & $\begin{array}{l}1.55 \\
(1.12,2.16)\end{array}$ & $\begin{array}{l}1.49 \\
(1.05,2.17)\end{array}$ & $\begin{array}{l}1.43 \\
(1.07,2.10)\end{array}$ & $\begin{array}{l}1.34 \\
(0.99,1.93)\end{array}$ & $\bowtie 0.001$ \\
\hline Creatinine, umol/L & $78(69,87)$ & $79(70,89)$ & $81(71.5,91)$ & $83(72,96)$ & $\varangle 0.001$ \\
\hline Peak cTNI, ng/ml & $\begin{array}{l}0.084 \\
(0.008,0.62)\end{array}$ & $\begin{array}{l}0.67 \\
(0.12,4.24)\end{array}$ & $\begin{array}{l}4.28 \\
(0.95,12.25)\end{array}$ & $\begin{array}{l}8.21 \\
(2.42,23.60)\end{array}$ & $\varangle 0.001$ \\
\hline Peak CK-MB, ng/ml & $\begin{array}{l}2.79 \\
(1.33,14.35)\end{array}$ & $\begin{array}{l}10.85 \\
(3.16,53.53)\end{array}$ & $\begin{array}{l}48.5 \\
(9.91,146.0)\end{array}$ & $\begin{array}{l}79.1(17.0- \\
197.0)\end{array}$ & $\varangle 0.001$ \\
\hline hs-CRP, ng/ml & $\begin{array}{l}1.28 \\
(0.60,3.37)\end{array}$ & $\begin{array}{l}1.83 \\
(0.73,4.88)\end{array}$ & $\begin{array}{l}3.13 \\
(1.28,9.29)\end{array}$ & $\begin{array}{l}7.62 \\
(2.52,18.45)\end{array}$ & $\bigotimes 0.001$ \\
\hline $\begin{array}{l}\text { three-vessel disease, } \\
\text { n (\%) }\end{array}$ & 712 (71.5) & 781 (78.4) & $793(79.6)$ & $806(81.0)$ & $\varangle 0.001$ \\
\hline \multicolumn{6}{|l|}{ Echocardiography } \\
\hline LAD, cm & $3.60 \pm 0.36$ & $3.69 \pm 0.40$ & $3.73 \pm 0.43$ & $3.76 \pm 0.50$ & $\varangle 0.001$ \\
\hline LVEDD, cm & $5.01 \pm 0.40$ & $5.0 \pm 0.43$ & $5.06 \pm 0.43$ & $5.09 \pm 0.47$ & $\varangle 0.001$ \\
\hline LVEF, \% & $68 \%(65,71 \%)$ & $67 \%(63,71 \%)$ & $\begin{array}{l}65 \%(60 \\
68 \%)\end{array}$ & $61 \%(55,66 \%)$ & $\nabla 0.001$ \\
\hline \multicolumn{6}{|c|}{ Medication during follow-up, $n$ (\%) } \\
\hline Aspirin & 882 (88.3) & 879 (88.3) & 863 (86.6) & 850 (85.4) & 0.164 \\
\hline $\begin{array}{l}\text { P2Y12 receptor } \\
\text { antagonist* }\end{array}$ & 922 (92.3) & $930(93.4)$ & 949 (95.3) & 941 (94.56) & 0.028 \\
\hline \multicolumn{6}{|c|}{$\begin{array}{l}\text { NT-proBNP, N-Terminal pro-brain natriuretic peptide; BP, blood pressure; STEMI, ST-elevation } \\
\text { myocardial infarction; NSTEMI, non-ST-segment elevation myocardial infarction; UAP, unstable angina } \\
\text { pectoris; LDL-C, low-density lipoprotein cholesterol; cTNI, cardiac troponin I; CK-MB, creatine kinase- } \\
\text { MB; hs-CRP, high-sensitivity C-reactive protein; LAD, left atrium diameter; LVEDD, left ventricular end- } \\
\text { diastolic diameter; ACEl, angiotensin-converting enzyme inhibitors, LVEF, left ventricular ejection } \\
\text { fraction; ARB, angiotensin receptor blockers. }\end{array}$} \\
\hline
\end{tabular}




\begin{tabular}{|c|c|c|c|c|c|}
\hline Variable & $\begin{array}{l}\text { Q1 } \\
(n=999)\end{array}$ & $\begin{array}{l}\text { Q2 } \\
(n=996)\end{array}$ & $\begin{array}{l}\text { Q3 } \\
(n=996)\end{array}$ & $\begin{array}{l}\text { Q4 } \\
(n=995)\end{array}$ & $\begin{array}{l}P \\
\text { value }\end{array}$ \\
\hline$\beta$-blocker & $617(61.8)$ & $614(61.8)$ & $628(63.1)$ & $628(63.1)$ & 0.847 \\
\hline ACEI/ARB & $459(45.9)$ & $546(54.8)$ & $560(56.2)$ & $574(57.7)$ & $\nabla 0.001$ \\
\hline Statin & $886(88.7)$ & $864(86.7)$ & $872(87.6)$ & $857(86.1)$ & 0.349 \\
\hline \multicolumn{6}{|c|}{$\begin{array}{l}\text { NT-proBNP, N-Terminal pro-brain natriuretic peptide; BP, blood pressure; STEMI, ST-elevation } \\
\text { myocardial infarction; NSTEMI, non-ST-segment elevation myocardial infarction; UAP, unstable angina } \\
\text { pectoris; LDL-C, low-density lipoprotein cholesterol; cTNI, cardiac troponin I; CK-MB, creatine kinase- } \\
\text { MB; hs-CRP, high-sensitivity C-reactive protein; LAD, left atrium diameter; LVEDD, left ventricular end- } \\
\text { diastolic diameter; ACEI, angiotensin-converting enzyme inhibitors, LVEF, left ventricular ejection } \\
\text { fraction; ARB, angiotensin receptor blockers. }\end{array}$} \\
\hline \multicolumn{6}{|c|}{ *P2Y12 receptor antagonist within 12 months after $\mathrm{PCl}$} \\
\hline
\end{tabular}

Age, heart rate, proportion of patients with STEMI and three-coronary artery lesion increased significantly in the higher quartile groups (Q3-Q4, $P<0.001$ ), while Body mass index (BMI), diastolic blood pressures (BP), proportion of male and UAP decreased significantly $(P<0.001)$. LVEF declined significantly with the increase of NT-proBNP levels in the Q3-Q4 groups $(P<0.001)$ although all subjects had LVEF $\geq 50 \%$. Fasting glucose levels at admission were significantly higher in the Q3-Q4 group $(P<0.001)$, but $\mathrm{Hb} 1 \mathrm{C}$ levels were not different in the 4 groups $(P=0.567)$.

As expected, peak troponin I, CK-MB, and hs-CRP were higher in the higher NT-proBNP quartile groups (Q3Q4, $P<0.001)$, also creatinine was higher in the Q3-Q4 groups $(P<0.001)$. Figure $2 \mathrm{~A}, 2 \mathrm{~B}$ and $2 \mathrm{C}$ illustrate significant positive correlations of Log [NT-proBNP] values and peak cTnl $(r=0.418, P<0.001)$, hs-CRP ( $r$ $=0.397, P<0.001)$, and LVEDD $(r=0.075, P<0.001)$, respectively. Figure 2D shows negative correlation between Log [NT-proBNP] values and LVEF $(r=-0.426, P<0.001)$ in this study cohort.

Not surprisingly, the length of hospital stay was longer in the Q3 and Q4 groups $(P<0.001)$.Post hospital discharge, $\mathrm{P} 2 \mathrm{Y} 12$ receptor antagonist $(P=0.028)$ and angiotensin-converting-enzyme inhibitors $(\mathrm{ACEI})+$ angiotensin receptor blockers(ARB) $(P<0.001)$ were used more frequently in the Q3-Q4 groups, and there were no significant differences in the usage of aspirin $(P=0.164), \beta$-blocker $(P=0.847)$ and statin $(P=$ 0.349) among Q1-Q4 groups.

\section{Incidence of MACCE and NT-proBNP}

Overall, the incidence of MACCE was $11.9 \%(n=476)$ during the median follow-up period of 35 (IQR: $23,48)$ months. There were significant differences in the occurrence of primary and secondary endpoints among Q1-Q4 groups: composite MACCE $(5.6 \%, 9.1 \%, 13.0 \%, 20.1 \%, P<0.001)$, all-cause mortality (1.0\%, $2.5 \%, 4.1 \%, 8.4 \%, P<0.001)$, CV death $(0.4 \%, 0.9 \%, 1.9 \%, 4.6 \%, P<0.001)$, nonfatal $\mathrm{Ml}(2.0 \%, 3.4 \%, 4.8 \%$, $6.2 \%, P<0.001)$ and HFRH $(1.5 \%, 2.3 \%, 4.1 \%, 5.9 \%, P<0.001)$. However, there was no significant difference in stroke in the 4 groups $(1.4 \%, 1.4 \%, 1.3 \%, 2.1 \%, P=0.438)$. These differences are also illustrated in detail by Kaplan-Meier curves (Figure 3A-3F). 


\section{Prediction of MACCE}

As detailed in Table 2, the univariate Cox proportional hazards model including variables listed in Table 1 identified that NT-proBNP, age, sex, BMI, heart rate, systolic BP, history of hypertension and diabetes mellitus, smoking, prior stroke, fasting glucose, creatinine, diagnosis of $\mathrm{Ml}$, three-vessel disease, length of stay in hospital were significantly correlated to the risk of composite of MACCE $(P<0.05)$.Then, multivariate Cox regression detected that higher NT-proBNP was a significant and independent predictor of increased risk of MACCE in this study population. For every $337 \mathrm{pg} / \mathrm{ml}$ (median) increase in NT-proBNP, the risk of MACCE went up 1.02-fold $(95 \% \mathrm{Cl}, 1.013-1.026 ; P<0.001)$. Compared to Q1 group, hazard ratio $(\mathrm{HR})$ raised to $1.29(95 \% \mathrm{Cl}, 0.92-1.81 ; P=0.137)$ in $\mathrm{Q} 2$ group; $1.73(95 \% \mathrm{Cl}, 1.25-2.38 ; P=0.001)$ and 2.16 $(95 \% \mathrm{Cl}, 1.57-2.99 ; P<0.001)$ in Q3 and Q4 groups respectively. In addition, age, heart rate, history of hypertension and diabetes mellitus, three-vessel disease and length of stay in the hospital (Figure 4) were independently and significantly associated with increased risks of MACCE $(p<0.05)$. 
Table 2

Univariate Cox proportional hazards regression analyses for major adverse cardiovascular and cerebral event

\begin{tabular}{|c|c|c|c|}
\hline \multirow[t]{2}{*}{ Variable } & \multicolumn{3}{|c|}{ Univariate regression } \\
\hline & HR & $95 \% \mathrm{Cl}$ & $P$ value \\
\hline NT-proBNP (per 337 pg/mL) & 1.031 & $1.026-1.036$ & $\nabla 0.001$ \\
\hline \multicolumn{4}{|l|}{ NT-proBNP } \\
\hline Q1 & Reference & - & - \\
\hline Q2 & 1.554 & $1.114-2.168$ & 0.009 \\
\hline Q3 & 2.08 & $1.520-2.847$ & $\otimes 0.001$ \\
\hline Q4 & 3.292 & $2.447-4.430$ & $\bigotimes 0.001$ \\
\hline Age, years & 1.043 & $1.034-1.052$ & $\otimes 0.001$ \\
\hline Male & 0.821 & $0.680-0.993$ & 0.042 \\
\hline Body mass index, $\mathrm{kg} / \mathrm{m}^{2}$ & 0.970 & $0.944-0.997$ & 0.028 \\
\hline Systolic BP, mmHg & 1.006 & $1.002-1.010$ & 0.008 \\
\hline Diastolic BP, mmHg & 0.997 & $0.989-1.004$ & 0.386 \\
\hline Heart rate, beats/min & 1.012 & $1.005-1.019$ & $\llbracket 0.001$ \\
\hline Hypertension & 1.445 & $1.177-1.773$ & $\llbracket 0.001$ \\
\hline Diabetes mellitus & 1.413 & $1.178-1.696$ & $\llbracket 0.001$ \\
\hline Dyslipidemia & 0.890 & $0.741-1.069$ & 0.213 \\
\hline Prior stroke & 1.756 & $1.414-2.179$ & $\bigotimes 0.001$ \\
\hline Smoking & 0.786 & $0.652-0.947$ & 0.011 \\
\hline UAP & Reference & - & - \\
\hline STEMI & 1.381 & $1.117-1.708$ & 0.003 \\
\hline NSTEMI & 1.512 & $1.210-1.889$ & $\otimes 0.001$ \\
\hline Length of stay, days & 1.084 & $1.062-1.105$ & $\otimes 0.001$ \\
\hline
\end{tabular}

$\mathrm{HR}$, hazard ratio ; Cl, confidence interval; NT-proBNP, N-Terminal pro-brain natriuretic peptide; BP, blood pressure; STEMI, ST-elevation myocardial infarction; NSTEMI, non-ST-segment elevation myocardial infarction; UAP, unstable angina pectoris; LDL-C, low-density lipoprotein cholesterol; cTNI, cardiac troponin I; CK-MB, creatine kinase-MB; hs-CRP, high-sensitivity C-reactive protein; ACEl, angiotensinconverting enzyme inhibitors, ARB, angiotensin receptor blockers.

*P2Y12 receptor antagonist within 12 months after $\mathrm{PCl}$ 


\begin{tabular}{|c|c|c|c|}
\hline \multirow[t]{2}{*}{ Variable } & \multicolumn{3}{|c|}{ Univariate regression } \\
\hline & HR & $95 \% \mathrm{Cl}$ & $P$ value \\
\hline Fasting glucose, $\mathrm{mmol} / \mathrm{L}$ & 1.052 & $1.017-1.088$ & 0.003 \\
\hline Hemoglobin A1c, \% & 1.074 & $1.011-1.142$ & 0.02 \\
\hline Total cholesterol, mmol/L & 0.927 & $0.849-1.013$ & 0.095 \\
\hline LDL-C, mmol/L & 0.907 & $0.803-1.024$ & 0.116 \\
\hline Triglyceride, mmol/L & 0.930 & $0.857-1.008$ & 0.079 \\
\hline Creatinine, mmol/L & 1.010 & $1.005-1.014$ & $\varangle 0.001$ \\
\hline Peak cTNI, ng/ml & 1.001 & $0.994-1.009$ & 0.726 \\
\hline Peak CK-MB, ng/ml & 1.000 & $0.999-1.001$ & 0.629 \\
\hline hs-CRP, ng/ml & 1.006 & $0.998-1.015$ & 0.155 \\
\hline three-vessel disease & 1.794 & $1.381-2.331$ & $\nabla 0.001$ \\
\hline Aspirin & 1.105 & $0.845-1.446$ & 0.167 \\
\hline P2Y12 receptor antagonist* & 0.574 & $0.415-0.794$ & 0.001 \\
\hline$\beta$-blocker & 0.982 & $0.816-1.181$ & 0.847 \\
\hline ARB/ACEI & 1.180 & $0.984-1.416$ & 0.073 \\
\hline Statin & 0.824 & $0.644-1.054$ & 0.124 \\
\hline \multicolumn{4}{|c|}{$\begin{array}{l}\text { HR, hazard ratio ; Cl, confidence interval; NT-proBNP, N-Terminal pro-brain natriuretic peptide; BP, bloo } \\
\text { pressure; STEMI, ST-elevation myocardial infarction; NSTEMI, non-ST-segment elevation myocardial } \\
\text { infarction; UAP, unstable angina pectoris; LDL-C, low-density lipoprotein cholesterol; cTNI, cardiac } \\
\text { troponin I; CK-MB, creatine kinase-MB; hs-CRP, high-sensitivity C-reactive protein; ACEI, angiotensin- } \\
\text { converting enzyme inhibitors, ARB, angiotensin receptor blockers. }\end{array}$} \\
\hline \multicolumn{4}{|c|}{ *P2Y12 receptor antagonist within 12 months after $\mathrm{PCl}$} \\
\hline
\end{tabular}

\section{Improved prediction of MACCE}

ROCs were generated using the TIMI risk score and NT-proBNP added to TIMI risk score. As shown in Figure 5, AUC improved slightly, but significantly for predicting MACCE with TIMI+NT-proBNP versus TIMI risk score alone [0.70 (95\% Cl, 0.69-0.72) vs.0.69 (95\% Cl, 0.67-0.70); $P=0.0012]$, respectively. There was a larger AUC improvement for CV death with TIMI+NT-proBNP versus TIMI risk score alone $[0.76(95 \% \mathrm{Cl}$, $0.75-0.78)$ vs. $0.72(95 \% \mathrm{Cl}, 0.71-0.73)$ with TIMI risk score, $P=0.0008]$. In addition, AUC also improved for prediction of HF requiring hospitalization with TIMI+NT-proBNP compared to TIMI risk score alone [0.68 (95\% Cl, 0.66-0.69) vs. $0.66(95 \% \mathrm{Cl}, 0.64-0.67) ; P=0.0017]$.

\section{Discussion}


Advancements in $\mathrm{PCl}$ and medical management in the past several decades have significantly improved the clinical outcomes in patients with $\mathrm{ACS}^{15,16}$. A successful $\mathrm{PCl}$ is achieved when residual diameter stenosis severity is reduced to $<30 \%$ in all treated lesions without an in-hospital major adverse cardia event including death, $\mathrm{Ml}$ or repeat coronary revascularization of the target lesions ${ }^{17}$. It is also important for a better outcome if LVEF remains normal at discharge ${ }^{3-5}$. However, MACCE continue to occur in patients undergoing a successful PCl and with normal LVEF. The current study in 3986 ACS patients demonstrated that $12 \%$ developed MACCE during a median of 35 months post successful PCI. Importantly, the study found that higher NT-proBNP during the initial ACS hospitalization was independently and significantly associated with increased risk of subsequent MACCE including all-cause death, non-fatal MI, stroke, and HFRH. Furthermore, the predictions of MACCE, CV death and HFRH were significantly improved when adding NT-proBNP to the TIMI risk score.

NT-proBNP is well established biomarker for diagnosis and prognosis of HF ${ }^{18-20}$. In the acute HF setting, the PRIDE study demonstrated that the sensitivity and specificity of NT-proBNP were $90 \%$ and $85 \%$ for diagnosis using a cut-point of $900 \mathrm{pg} / \mathrm{ml}$ in emergency department ${ }^{18}$. Furthermore, NT-proBNP level< $300 \mathrm{pg} / \mathrm{ml}$ was optimal for ruling out acute HF with a negative predictive value of $99 \%$. Study from Berin $\mathrm{R}$ et al. also displayed that serum NT-proBNP was the strongest predictor of long-term mortality in patients with chronic HF (HR 3.76, 95\% Cl, 1.20-11.80; $P=0.008)^{20}$. In addition, higher NT-proBNP levels were strongly associated with increased risks of all-cause death, cardiac death, and MACCE $(P<0.01)$ in CAD patients with three-vessel disease ${ }^{21}$ and has been considered as a useful biomarker for risk stratification and therapeutic decision-making. The present study found that there was a wide range of NT-proBNP values in the ACS patients undergoing successful $\mathrm{PCl}$ and with LVEF $\geq 50 \%$. The median levels were $56 \mathrm{pg} / \mathrm{ml}$ and $2706 \mathrm{pg} / \mathrm{ml}$ in the lowest and highest NT-proBNP quartile groups. More importantly, incidence of MACCE showed stepwise increase from low to high quartiles (Q1-Q4) $(5.6 \%$, $9.1 \%, 13.0 \%, 20.1 \%, P<0.001)$. Similar significant trends were also detected in the incidences of all-cause mortality, cardiovascular death, nonfatal MI and HFRH (all with $P<0.001$ ), but, stroke. Multivariate analysis revealed that the risk of MACCE increased independently by 1.02 -fold $(95 \% \mathrm{Cl}, 1.01-1.03 ; P<$ 0.001 ) for every $337 \mathrm{pg} / \mathrm{ml}$ increase in NT-proBNP. These findings suggest that NT-proBNP, as a common and routine parameter assessed during ACS hospitalization, can effectively predict long-term (a median of 35 months) adverse outcomes after successful PCI for ACS and with LVEF $\geq 50 \%$.

In the current study, multivariate Cox regression analysis also identified clinical factors such as age, heart rate, history of hypertension and diabetes mellitus, three-vessel disease and length of stay in the hospital, in addition to NT-proBNP (Fig. 5), independently predicted the risks of subsequent MACCE (all $P<0.05$ ). The existing TIMI risk score using mainly clinical factors has been helpful in predicting outcomes in the ACS population $2,22,23$. We applied TIMI risk score to our ACS patients who received successful PCI and had normal LVEF and found that AUCs were 0.69 for MACCE, 0.72 for CV death and 0.66 for HFRH. Our study found significantly improved AUCs, 0.70 for MACCE, 0.76 for CV death and 0.68 for HFRH when NTproBNP was added to the TIMI risk score. These results showed an improved prognostic value of adding NT-proBNP to the existing TIMI risk score for subsequent MACCE in the ACS patients with successful PCI 
and normal LVEF. Future studies are needed to investigate whether therapies guided by further risk stratification using NT-proBNP can improve clinical outcomes.

Previous studies have demonstrated that increased levels of NT-proBNP were associated with myocardial ischemic injury, inflammation and remodeling during $\mathrm{ACS}^{24-26}$. Indeed, our study found significant positive correlations between Log (NT-proBNP) values and peak cTnl $(r=0.418, P<0.001)$ and between Log (NT-proBNP) and hs-CRP $(r=0.397, P<0.001)$. It is well known that reduced LVEF due to myocardial remodeling during and post ischemic injury predicts cardiovascular morbidity and mortality ${ }^{3-5}$. Previous studies also showed that NT-proBNP is marker of myocardial remodeling ${ }^{7,26,27}$. Hendriks and colleagues $^{26}$ suggested that NT-proBNP was a better predictor of LV volume indices which were the marks of left ventricular remodeling. Within their multivariable model, NT-proBNP was strong predictor for the LV end-diastolic volume index (LVEDVi), end-systolic volume index (LVESVi) and mass index (LVMi) as assessed by cardiac magnetic resonance imaging in patients 4 months after STEMI (LVEF $54 \pm 9 \%$ ). In the current study, we found a significant negative correlation between Log [NT-proBNP] values and echo assessed LVEF $(r=-0.426, P<0.001)$ in patients with normal LVEF. The correlation between Log (NTproBNP) values and LVEDD was significant, but modest $(r=0.075, P<0.001)$. These data represent that myocardial remodeling is occurring in the subclinical state before reaching reduced LVEF and NT-proBNP is a useful marker in ACS patients with normal LVEF.

\section{Conclusions}

In conclusion, $12 \%$ of ACS patients who had successful PCl and normal LVEF at hospital discharge developed subsequent MACCE during 35 months of follow-up. Higher levels of NT-proBNP was an independent predictor of increased risk of MACCE and improved the prediction of MACCE in addition to the existing TIMI risk score in this population.

\section{List Of Abbreviations}

NT-proBNP: N-Terminal pro-brain natriuretic peptide; MACCE: major adverse cardiovascular and cerebral event; PCl: percutaneous coronary intervention; ACS: acute coronary syndrome; MI: myocardial infarction; LVEF: left ventricular ejection fraction; Q: quartile; HFRH: heart failure requiring hospitalization; AUC: area under the curve; $\mathrm{CV}$ : cardiovascular; TIMI: thrombolysis in myocardial infarction; echo: echocardiography; STEMI: ST-segment elevation myocardial infarction; NSTEMI: non-ST-segment elevation myocardial infarction; UAP: unstable angina pectoris; ECG: electrocardiogram; HF: heart failure; cTnl: cardiac troponin I; CK-MB: creatine kinase-MB; LAD: left atrium diameter; LVEDD: left ventricular end-diastolic diameter; CT: computed tomography; IQR: interquartile range; HR: hazard ratios; Cl: confidence intervals; ROC: receiver operating characteristic; BMI: body mass index; BP: blood pressures; ACEl: angiotensin-converting enzyme inhibitors; ARB: angiotensin receptor blockers; LVEDVi: left ventricular end-diastolic volume index; LVESVi: left ventricular end-systolic volume index; LVMi: left ventricular mass index; LDL-C, low-density lipoprotein cholesterol; hs-CRP, high-sensitivity C-reactive protein. 


\section{Declarations}

\section{Ethics approval and consent to participate}

The study data collections were approved by the Institutional Ethics Committee of

Beijing Friendship Hospital affiliated to Capital Medical University. The study was also in accordance with the 1964 Declaration of Helsinki and its later amendments or

comparable ethical standards.

\section{Consent for publication}

Not applicable.

\section{Availability of data and materials}

The datasets used and/or analyzed during the current study are available from the corresponding author on reasonable request.

\section{Competing interests}

The authors declare that they have no competing interests.

\section{Funding}

Not applicable.

\section{Authors' contributions}

JLW and SMZ performed study, statistical analysis and wrote manuscript. HWL and HC provided support, designed study and reviewed manuscript. CYG participated in study data collection. XQZ designed study and revised manuscript. All authors read and approved the final manuscript.

\section{Acknowledgements}

We gratefully acknowledge the contributions of all staffs who work on the CBD Bank.

\section{References}

1. Roffi M, Patrono C, Collet JP, et al. 2015 ESC Guidelines for the management of acute coronary syndromes in patients presenting without persistent ST-segment elevation: Task Force for the Management of Acute Coronary Syndromes in Patients Presenting without Persistent ST-Segment Elevation of the European Society of Cardiology (ESC). Eur Heart J. 2016;37:267-315. 
2. Antman EM, Cohen M, Bernink PJ, et al. The TIMI risk score for unstable angina/non-ST elevation MI: A method for prognostication and therapeutic decision making. JAMA. 2000;284:835-42.

3. Breathett K, Allen LA, Udelson J, Davis G, Bristow M. Changes in Left Ventricular Ejection Fraction Predict Survival and Hospitalization in Heart Failure With Reduced Ejection Fraction. Circ Heart Fail. 2016;9:e002962.

4. Kiris T, Avci E, Celik A. Combined value of left ventricular ejection fraction and the Model for EndStage Liver Disease (MELD) score for predicting mortality in patients with acute coronary syndrome who were undergoing percutaneous coronary intervention. BMC Cardiovasc Disord. 2018;18:44.

5. Sladojevic M, Sladojevic S, Culibrk D, Tadic S, Jung R. Echocardiographic parameters as predictors of in-hospital mortality in patients with acute coronary syndrome undergoing percutaneous coronary intervention. ScientificWorldJournal. 2014;2014:818365.

6. Perelshtein Brezinov O, Klempfner R, Zekry SB, Goldenberg I, Kuperstein R. Prognostic value of ejection fraction in patients admitted with acute coronary syndrome: A real world study. Med (Baltim). 2017;96:e6226.

7. Pan Y, Li D, Ma J, Shan L, Wei M. NT-proBNP test with improved accuracy for the diagnosis of chronic heart failure. Med (Baltim). 2017;96:e9181.

8. Xiaozhou H, Jie Z, Li Z, Liyan C. Predictive value of the serum level of N-terminal pro-brain natriuretic peptide and high-sensitivity C-reactive protein in left ventricular remodeling after acute myocardial infarction. J Clin Lab Anal. 2006;20:19-22.

9. de Lemos JA, McGuire DK, Drazner MH. B-type natriuretic peptide in cardiovascular disease. Lancet. 2003;362:316-22.

10. Oremus $M$, Don-Wauchope A, McKelvie R, et al. BNP and NT-proBNP as prognostic markers in persons with chronic stable heart failure. Heart Fail Rev. 2014;19:471-505.

11. Zdravkovic V, Mladenovic V, Colic M, et al. NT-proBNP for prognostic and diagnostic evaluation in patients with acute coronary syndromes. Kardiol Pol. 2013;71:472-9.

12. Heeschen $\mathrm{C}$, Hamm CW, Mitrovic V, Lantelme NH, White HD. Platelet Receptor Inhibition in Ischemic Syndrome Management I. N-terminal pro-B-type natriuretic peptide levels for dynamic risk stratification of patients with acute coronary syndromes. Circulation. 2004;110:3206-12.

13. Anderson JL, Adams CD, Antman EM, et al. ACC/AHA 2007 guidelines for the management of patients with unstable angina/non-ST-Elevation myocardial infarction: a report of the American College of Cardiology/American Heart Association Task Force on Practice Guidelines (Writing Committee to Revise the 2002 Guidelines for the Management of Patients With Unstable Angina/Non-ST-Elevation Myocardial Infarction) developed in collaboration with the American College of Emergency Physicians, the Society for Cardiovascular Angiography and Interventions, and the Society of Thoracic Surgeons endorsed by the American Association of Cardiovascular and Pulmonary Rehabilitation and the Society for Academic Emergency Medicine. J Am Coll Cardiol. 2007; $50: e 1-157$. 
14. Neumann FJ, Sousa-Uva M, Ahlsson A, et al. 2018 ESC/EACTS Guidelines on myocardial revascularization. Eur Heart J. 2019;40:87-165.

15. Ottervanger JP, Armstrong P, Barnathan ES, et al. Association of revascularisation with low mortality in non-ST elevation acute coronary syndrome, a report from GUSTO IV-ACS. Eur Heart J. 2004;25:1494-501.

16. Lehmann R, Fichtlscherer S, Schachinger V, et al. Complete revascularization in patients undergoing multivessel $\mathrm{PCl}$ is an independent predictor of improved long-term survival. J Interv Cardiol. 2010;23:256-63.

17. Levine GN, Bates ER, Blankenship JC, et al. 2011 ACCF/AHA/SCAI Guideline for Percutaneous Coronary Intervention. A report of the American College of Cardiology Foundation/American Heart Association Task Force on Practice Guidelines and the Society for Cardiovascular Angiography and Interventions. J Am Coll Cardiol. 2011;58:e44-122.

18. Januzzi JL Jr, Camargo CA, Anwaruddin S, et al. The N-terminal Pro-BNP investigation of dyspnea in the emergency department (PRIDE) study. Am J Cardiol. 2005;95:948-54.

19. Januzzi JL, van Kimmenade R, Lainchbury J, et al. NT-proBNP testing for diagnosis and short-term prognosis in acute destabilized heart failure: an international pooled analysis of 1256 patients: the International Collaborative of NT-proBNP Study. Eur Heart J. 2006;27:330-7.

20. Berin R, Zafrir B, Salman N, Amir O. Single measurement of serum N-terminal pro-brain natriuretic peptide: the best predictor of long-term mortality in patients with chronic systolic heart failure. Eur $\mathrm{J}$ Intern Med. 2014;25:458-62.

21. Zhang C, Jiang L, Xu L, et al. Implications of N-terminal pro-B-type natriuretic peptide in patients with three-vessel disease. Eur Heart J. 2019;40:3397-405.

22. Karabag Y, Cagdas M, Rencuzogullari I, et al. Comparison of SYNTAX score II efficacy with SYNTAX score and TIMI risk score for predicting in-hospital and long-term mortality in patients with ST segment elevation myocardial infarction. Int J Cardiovasc Imaging. 2018;34:1165-75.

23. Schellings DA, Adiyaman A, Dambrink JE, et al. Predictive value of NT-proBNP for 30-day mortality in patients with non-ST-elevation acute coronary syndromes: a comparison with the GRACE and TIMI risk scores. Vasc Health Risk Manag. 2016;12:471-6.

24. Mayr A, Mair J, Schocke M, et al. Predictive value of NT-pro BNP after acute myocardial infarction: relation with acute and chronic infarct size and myocardial function. Int J Cardiol. 2011;147:118-23.

25. van Diepen S, Roe MT, Lopes RD, et al. Baseline NT-proBNP and biomarkers of inflammation and necrosis in patients with ST-segment elevation myocardial infarction: insights from the APEX-AMI trial. J Thromb Thrombolysis. 2012;34:106-13.

26. Hendriks T, Hartman MHT, Vlaar PJJ, et al. Predictors of left ventricular remodeling after ST-elevation myocardial infarction. Int J Cardiovasc Imaging. 2017;33:1415-23.

27. Nilsson JC, Groenning BA, Nielsen G, et al. Left ventricular remodeling in the first year after acute myocardial infarction and the predictive value of $\mathrm{N}$-terminal pro brain natriuretic peptide. Am Heart $\mathrm{J}$. 2002;143:696-702. 
Figures

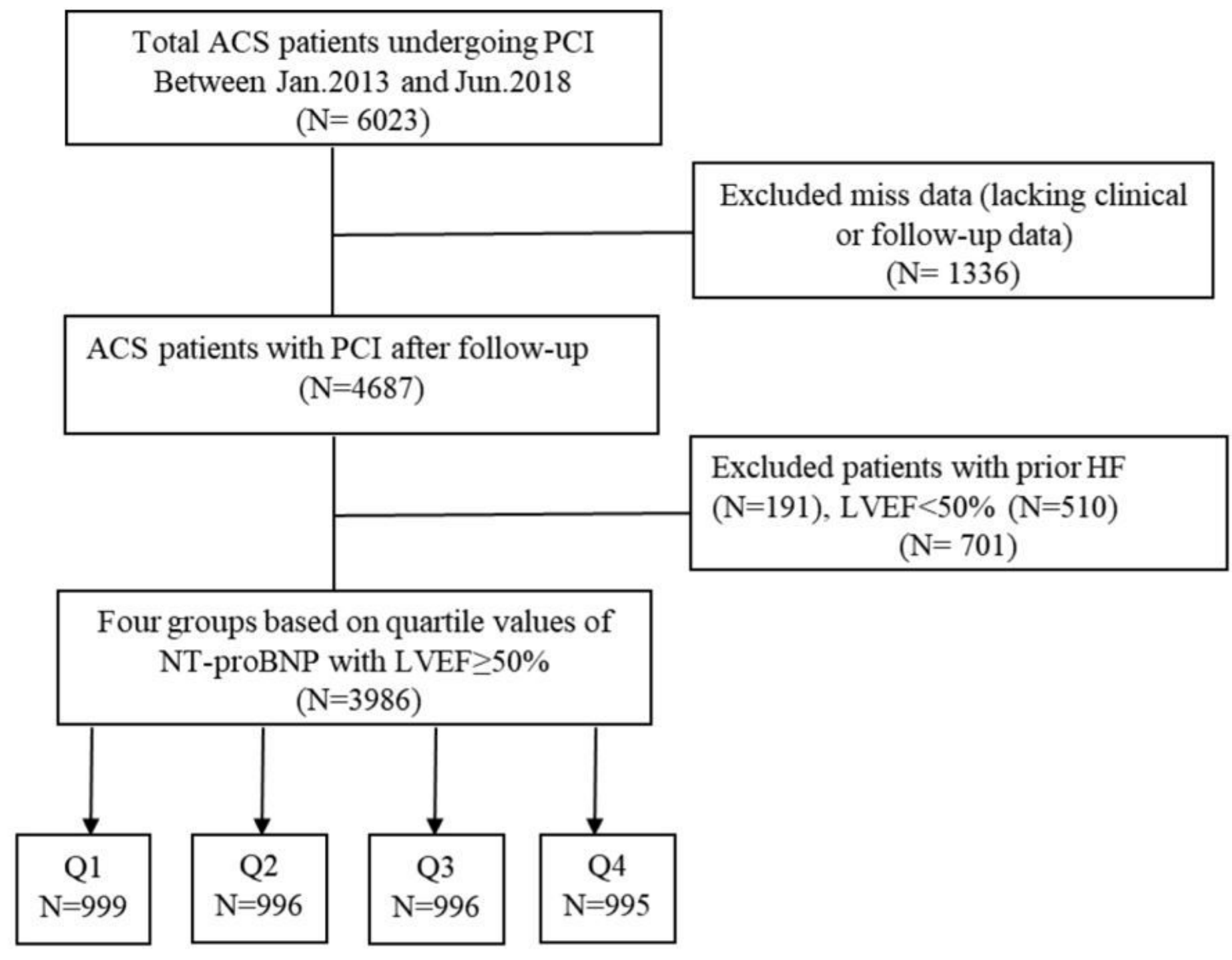

Figure 1

Patient selection flow. ACS, acute coronary syndrome; $\mathrm{PCl}$, percutaneous coronary intervention; LVEF, left ventricular ejection fraction; HF, heart failure; NT-proBNP, N-Terminal pro-brain natriuretic peptide. 

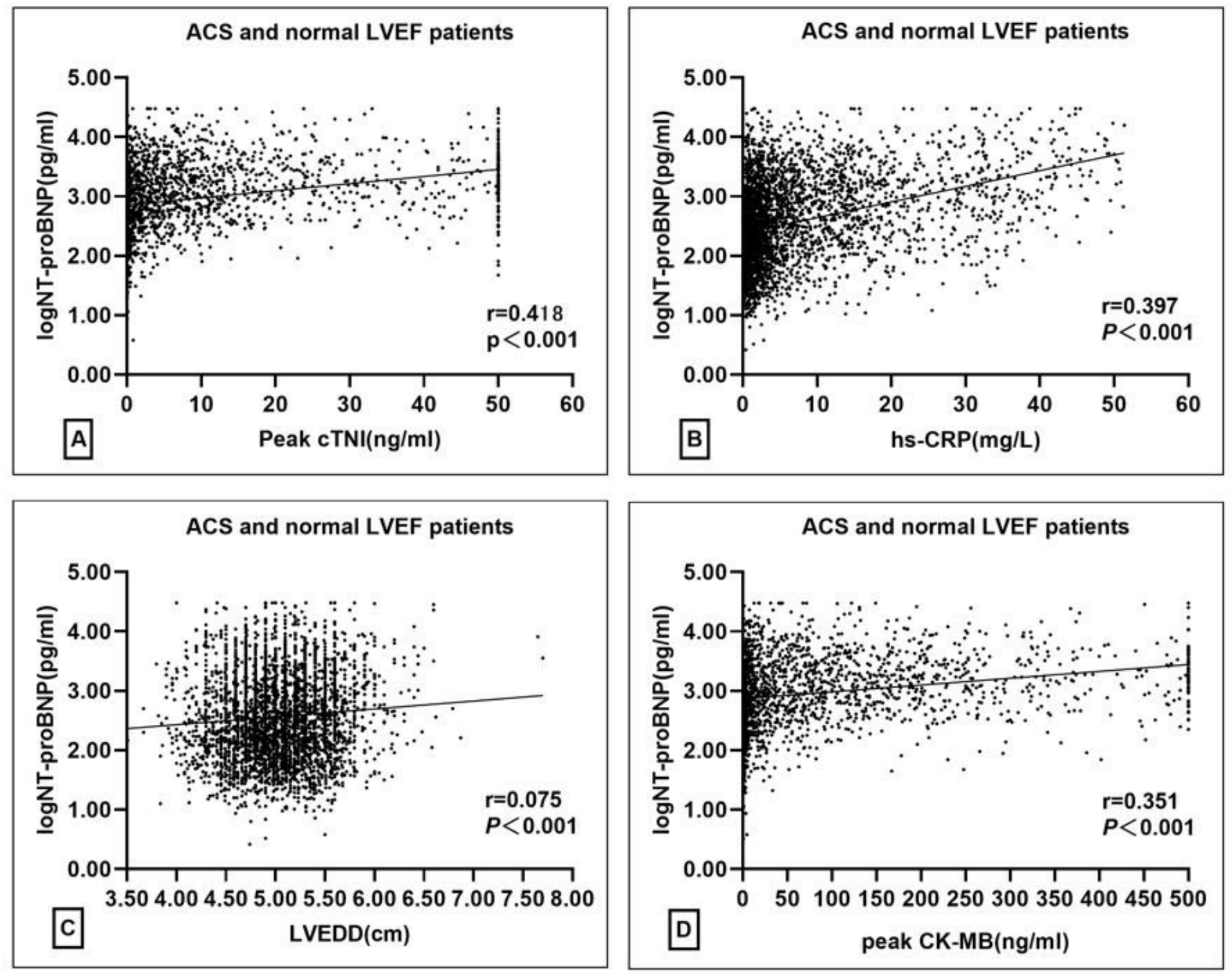

Figure 2

Significant positive correlation between Log [NT-proBNP] and peak cTNI, hs-CRP, LVEDD $(P<0.001, A, B$, C). Significant negative correlation between Log [NT-proBNP] and LVEF $(P<0.001, D)$. NT-proBNP, NTerminal pro-brain natriuretic peptide; cTNI, cardiac troponin I; hs-CRP, high-sensitivity C-reactive protein; LVEDD, left ventricular end-diastolic diameter; LVEF, left ventricular ejection fraction. 


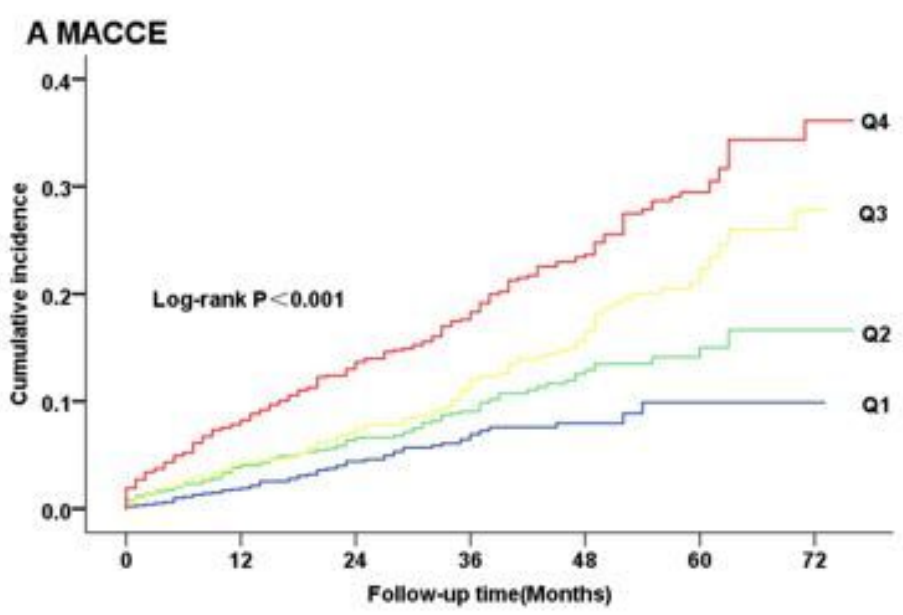

\section{B All-cause death}
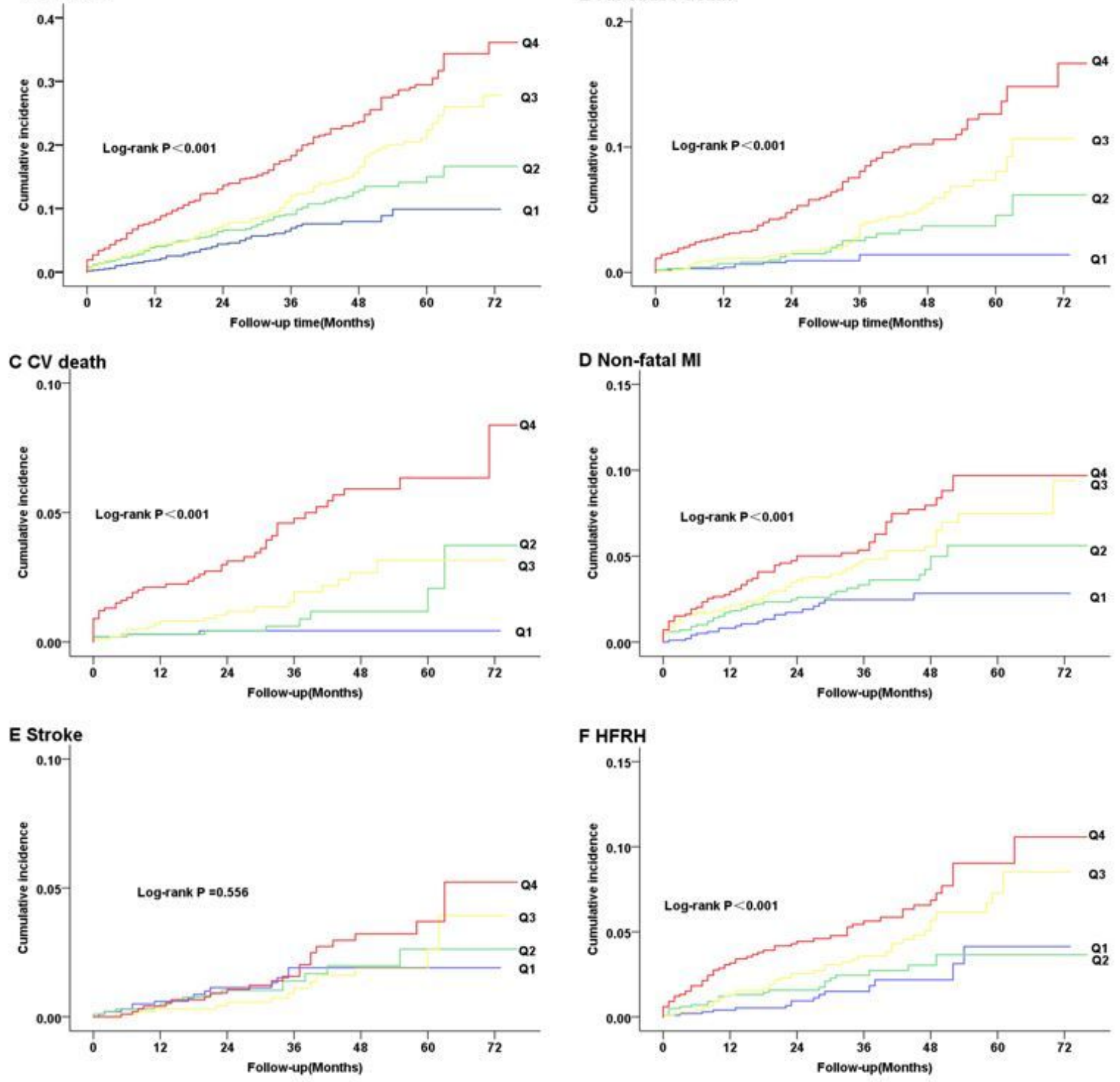

Figure 3

Kaplan-Meier curve analyses during follow-up according to NT-proBNP quartile groups. Cumulative incidence of MACCE (A), all-cause death (B), cardiovascular death (C), nonfatal MI (D), ischemic stroke $(E)$, and heart failure requiring hospitalization $(F)$. NT-proBNP, N-Terminal pro-brain natriuretic peptide; MACCE, major adverse cardiovascular and cerebral events. 


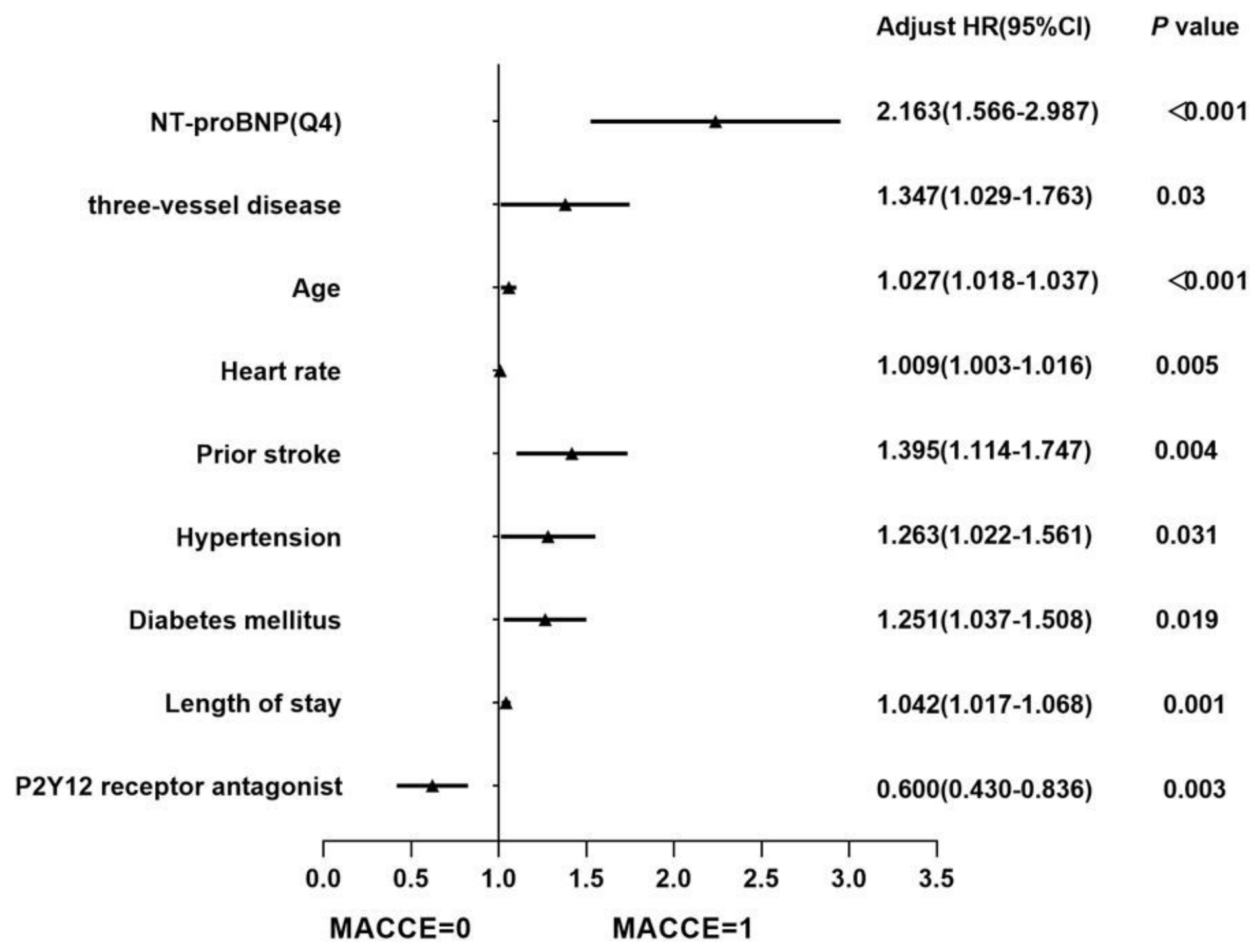

Figure 4

Factors independently associated with MACCE in multivariable Cox regression analysis. MACCE, major adverse cardiovascular and cerebral events. 

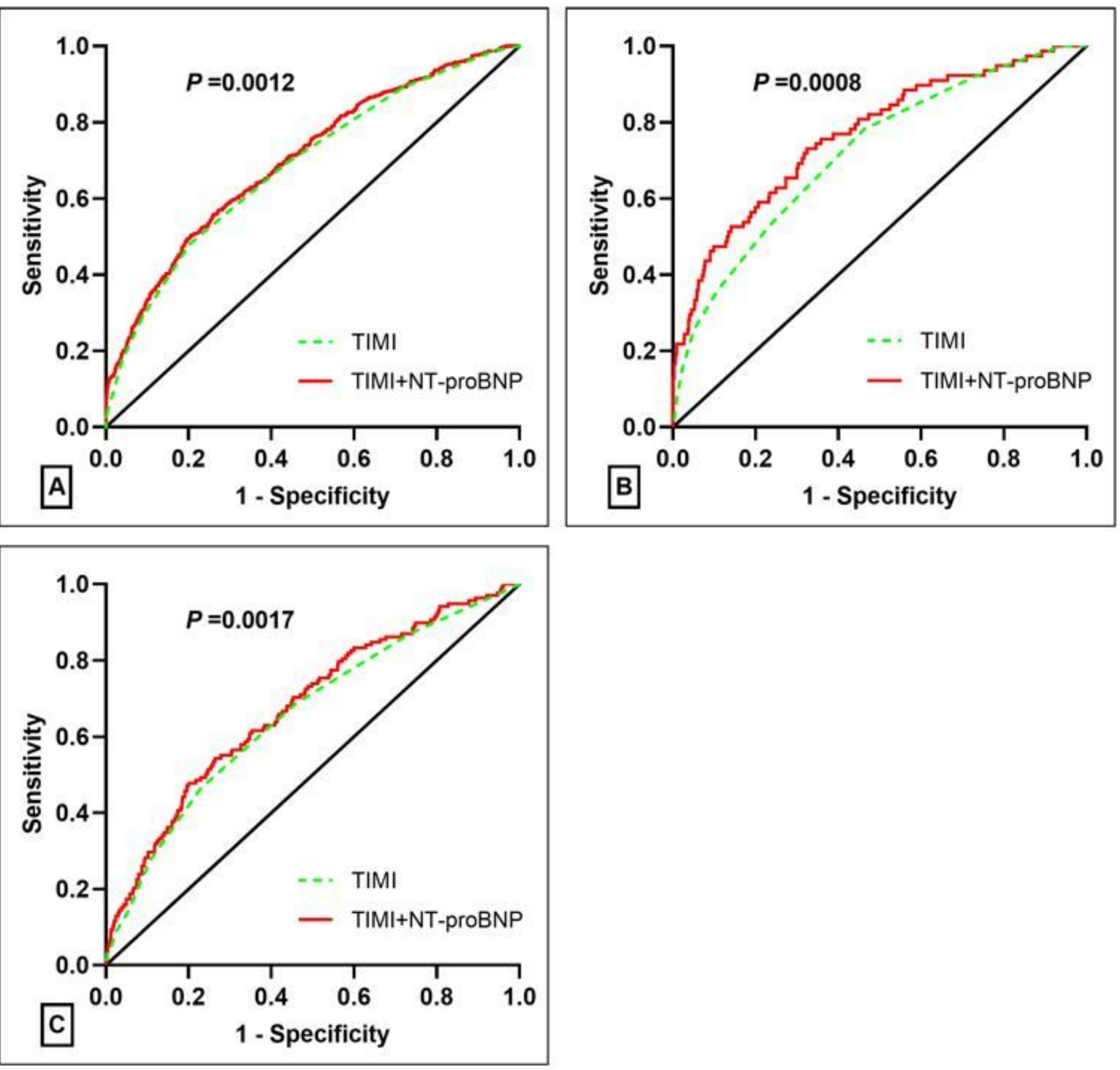

Figure 5

Receiver-operator characteristic curves for predictions of MACCE with TIMI risk score and TIMI +NTproBNP.(A) composite MACCE, 0.688 (95\% Cl, 0.673-0.702) vs. 0.702 (95\% Cl, 0.688-0.717; P =0.0012); (B) CV death: $0.720(95 \% \mathrm{Cl}, 0.706-0.734)$ vs. $0.765(95 \% \mathrm{Cl}, 0.752-0.779 ; \mathrm{P}=0.0008)$, and (C) HFRH, $0.656(95 \% \mathrm{Cl}, 0.641-0.671)$ vs.0.677 (95\% Cl, 0.662-0.692; $\mathrm{P}=0.0017)$. MACCE, major adverse cardiovascular and cerebral events; NT-proBNP, N-Terminal pro-brain natriuretic peptide; TIMI, thrombolysis in myocardial infarction; $\mathrm{HFRH}$, heart failure requiring hospitalization. 